\title{
PROTECCIÓN DEL MEDIO AMBIENTE Y PARTICIPACIÓN PÚBLICA: LA RECIENTE EVOLUCIÓN A NIVEL INTERNACIONAL Y EN ESPAÑA
}

Carmen Plaza Martín ${ }^{1}$

Facultad de Ciencias Jurídicas y Sociales de Toledo

\section{RESUMEN}

Este trabajo examina los avances en materia de participación pública que han tenido lugar recientemente en el ámbito de la protección del medio ambiente a nivel internacional y en España. En el veinte aniversario de la firma del Convenio sobre el acceso a la información, la participación del público en la toma de decisiones y el acceso a la justicia en materia de medio ambiente, firmado en Aarhus en 1998 bajo los auspicios de la Comisión Económica para Europa de Naciones Unidas, los progresos más relevantes han tenido lugar también en la esfera internacional. En 2018 se firma un nuevo acuerdo internacional que va a seguir sus pasos en la región de América Latina y el Caribe: el Acuerdo de Escazú. La primera parte de este trabajo se centra en una comparación entre las disposiciones en materia de participación pública de estos dos instrumentos internacionales. En cuanto a los novedades en España, la segunda parte de este trabajo examina las modificaciones operadas en materia de participación pública en la Ley de evaluación de impacto ambiental así como los retos pendientes para avanzar hacia una participación efectiva. Finalmente, en la tercera parte, se da cuenta de algunas importantes resoluciones judiciales aplicando las disposiciones sobre participación pública en materia de medio ambiente. El análisis de estas novedades pone de relieve la creciente importancia del derecho de participación en materia de medio ambiente y de su tutela judicial efectiva. En la espera internacional, la comparación entre el Convenio de Aarhus y el Convenio de Escazú revela que si bien este último se inspira en la estructura y enfoque del primero, va a prestar especial atención a los problemas específicos de la región para la que se ha diseñado, como la

1Licenciada y Doctora en Derecho por la Universidad Complutense de Madrid (Premio Extraordinario de Doctorado), Magister en Derecho de la Unión por la misma Universidad, y LL.M. in European Community Law por la Universidad de Essex (Reino Unido), en donde se especializó en Derechos Humanos en Europa, y en la contribución del Derecho Laboral de la Comunidad Europea en la lucha contra la discriminación por razón de género. 
igualdad en el acceso a la participación pública, así como a retos actuales tales como el uso de las nuevas tecnologías como medio para ejercerlos. El Convenio de Aarhus es, sin embargo, algo más preciso en la delimitación de las condiciones de participación que han de respetar las autoridades públicas en la toma de decisiones relativas a ciertas actividades susceptibles de tener un impacto significativo en el medio ambiente.

Por lo que se refiere a los avances en España, a nivel normativo vienen impulsados - una vez más - por la obligación de transponer al ordenamiento nacional las modificaciones de la Directiva de Evaluación Ambiental de la Unión Europea. Finalmente, se advierte que los tribunales españoles siguen desarrollando una notable jurisprudencia - no exenta de algunas sombras - para hacer efectivo el derecho de participación.

Palabras clave: Acuerdo de Escazú; aplicación judicial; avances y retos; Convenio de Aarhus; evaluación de impacto ambiental; participación efectiva.

\section{ENVIRONMENTAL PROTECTION AND PUBLIC PARTICIPATION: THE RECENT EVOLUCION AT THE INTERNATIONAL} LEVEL AND IN SPAIN

\section{ABSTRACT}

This work examines the recent progress that has taken place in the field of public participation and environmental protection in the international arena and in Spain. In the twentieth anniversary of the Convention on access to information, public participation in decision-making and access to justice in environmental issues, signed in Aarhus in 1998, the most significant improvements have also taken place in the international arena. In 2018, several Latin American and Caribbean countries signed the Escazú Agreement on Access to Information, Public Participation and Access to Justice in Environmental Mattes. The first part of this article focuses a comparison of the provisions on public participation of these two international Agreements. As to the developments taking place in Spain, the second part examines the amendments to the provisions on public participation of the Environmental Impact Assessment Law. Finally, it gives account of the most relevant judicial decision regarding the application of the Aarhus Convention provisions on public participation. 
The analysis of these developments highlights the increasing importance of the right to public participation in environmental matters and of its effective judicial protection. At international level the comparison between the Aarhus Convention and the Escazú Agreement reveals, first, that the latter follows essentially the structure and approach of the former. However, it places special attention to the specific problems of the region, such as equal access to public participation, as well as to new global challenges, such as the use of new technologies in public participation. The Aarhus Convention, on the other hand, is more precise at delimiting the participation requirements that national authorities has to fulfill in the decision making process on certain environmental activities which might have a significant impact in the environment.

With regard to the legal developments in Spain, they have been driven - once more - by the obligation to transpose the amendments to the EU Environmental Impact Directive. As to the judicial application of the international and national provisions on public participation, Spanish courts are developing an important case law - no without shadows -, to make effective the right of participation on environmental matters.

Keywords: Aarhus Convention; effective participation; environmental impact assessment; Escazú Agreement; judicial application, improvement and challenges.

\section{INTRODUCCION}

Han transcurrido ya más de dos décadas desde que 39 países europeos firmaran en la ciudad danesa de Aarhus, bajo los auspicios de la Comisión Económica para Europa de Naciones Unidas, el Convenio sobre el acceso a la información, la participación del público en la toma de decisiones y el acceso a la justicia en materia de medio ambiente. El Convenio de Aarhus, considerado hasta la fecha como la iniciativa más ambiciosa para impulsar la democracia ambiental universal (BAN KI-MOON, 2014, p. 3; PRIEUR, 1999, p. 9), ha propiciado a lo largo de todos estos años notables cambios normativos en los Estados Parte, en la Unión Europea (desde que por Decisión 2005/370/CE del Consejo de 17 de febrero de 2005 se aprobara su celebración en nombre de la entonces Comunidad Europea), y en España (a raíz de su ratificación el 29 de diciembre de 2004, y como miembro de la Unión Europea). 
Este instrumento internacional, ratificado a fecha de hoy por 47 países de Europa y Asia, está siendo también un referente fundamental para la adopción de instrumentos internacionales similares en otras regiones de nuestro planeta. Este es el caso del reciente Acuerdo regional sobre el acceso a la información, la participación pública y el acceso a la justicia en asuntos ambientales en América Latina y el Caribe, firmado el 4 de marzo de 2018 en la ciudad costarricense de Escazú con el apoyo de la Comisión Económica de las Naciones Unidas para América Latina y el Caribe (CEPAL).

El Acuerdo de Escazú sigue el enfoque del Convenio de Aarhus basado en derechos subjetivos (los tres derechos denominados "de acceso": derecho de acceso a la información, a la participación pública y a la justicia en materia de medio ambiente), y en el fortalecimiento de los principios democráticos en aras de la protección del medio ambiente.

En materia de participación, en particular, se van a establecer exigencias y condiciones en gran medida similares a las del Convenio impulsado hace veinte años para Europa, si bien el Acuerdo de Escazú introduce también disposiciones novedosas - como en materia de participación en foros internacionales -, y va a reflejar en su articulado las necesidades y particularidades de la región Latinoamericana y del Caribe - en algunos puntos muy distintas de las del entorno europeo.

Por otra parte, en España se han modificado recientemente las disposiciones sobre participación pública que contine la Ley 21/2013 de Evaluación Ambiental (en adelante LEA) por la Ley 9/2018, de 5 de diciembre. Como se analiza más adelante, se trata de unas modificaciones limitadas a garantizar el uso de medios electrónicos en los trámites de información pública y consulta a las Administraciones Públicas y a las personas interesadas que regula esta Ley. Estas disposiciones se introducen con el fin de completar la adecuación del ordenamiento español a las disposiciones de la Directiva 2014/52/UE del Parlamento Europeo y del Consejo, de 16 de abril de 2014, por la que se modifica la Directiva 2011/92/UE, relativa a la evaluación de las repercusiones de determinados proyectos públicos y privados sobre el medio ambiente.

Finalmente, cabe destacar algunas decisiones judiciales relevantes resolviendo los recursosinterpuestos por organizaciones no gubernamentales denunciado la vulneración de las disposiciones sobre participación pública del Convenio de Aarhus y de la normativa nacional. 
En este marco, se analiza a continuación, en primer lugar, las disposiciones en materia de participación del Acuerdo de Escazú, comparándolas con las adoptadas hace veinte años por Convenio de Aarhus. En segundo lugar, se examina el marco normativo básico adoptado por el Estado español para cumplir con el Convenio, en qué medida las modificaciones introducidas recientemente en la Ley de Evaluación Ambiental por la Ley 9/2018 suponen realmente un avance, y los principales retos que se encuentra todavía nuestro estado para avanzar hacia una participación pública en defensa del medio ambiente más efectiva. Se examina, finalmente, las principales decisiones judiciales aplicando las disposiciones vigentes en este ámbito. Junto con el examen analítico de las disposiciones de los tratados internacionales en cuestión, y de las normas estatales adoptadas en este ámbito, se han identificado resoluciones judiciales reciente de especial interés para poner de manifiesto los avances y dificultades que encuentra la aplicación judicial de las disposiciones del Convenio de Aarhus por los Tribunales nacionales. Finalmente, se ha revisado las aportaciones doctrinales al estudio de esta materia.

\section{LAS DISPOSICIONES SOBRE PARTICIPACIÓN PÚBLICA DEL ACUERDO REGIONAL DE ESCAZÚ: PRINCIPALES NOVEDADES}

\subsection{El objeto y el enfoque del Convenio de Escazú}

El 4 de marzo de 2018, tras prácticamente seis años de negociaciones, se adoptó en Escazú (Costa Rica) el Acuerdo Regional sobre el Acceso a la Información, la Participación Pública y el Acceso a la Justicia en Asuntos Ambientales en América Latina y el Caribe.

El impulso para la negociación de este tratado tiene su origen inmediato en la Conferencia de las Naciones Unidas sobre el Desarrollo Sostenible (Río+20), en la que varios países de América Latina y el Caribe (Chile, Costa Rica, Jamaica, México, Panamá, Paraguay, Perú, República Dominicana y Uruguay) formularon una "Declaración sobre la Aplicación del Principio 10 de la Declaración de Rio". Se comprometían en ella a elaborar e implementar un Plan de Acción 2012-2014, con el apoyo de CEPAL como secretaría técnica, para avanzar en la consecución de un convenio regional u otro instrumento, solicitando a dicha organización 
un estudio sobre la situación, mejores prácticas y necesidades en materia de acceso a la información, a la participación y a la justicia en temas ambientales en América Latina y el Caribe (GÓMEZ PEÑA, 2018). Finalmente, tras su adopción, se abrió formalmente a la firma de los 33 países de América Latina y el Caribe el 27 de septiembre de 2018 en la Sede de las Naciones Unidas en Nueva York, haciéndolo coincidir con el Debate General Anual de la Asamblea General de las Naciones Unidas, y destacando así su importancia para la consecución de los Objetivos de Desarrollo Sostenible de la Agenda 2030.

El Acuerdo de Escazú tiene como objetivo "garantizar la implementación plena y efectiva en esta región de los derechos de acceso a la información ambiental, participación pública en los procesos de toma de decisiones ambientales y acceso a la justicia en asuntos ambientales, así como la creación y el fortalecimiento de las capacidades y la cooperación, contribuyendo a la protección del derecho de cada persona, de las generaciones presentes y futuras, a vivir en un medio ambiente sano y al desarrollo sostenible" (art. 1). Sigue así, al igual que el Convenio de Aarhus, un enfoque basado en derechos subjetivos, y en el fortalecimiento de los principios democráticos en aras de la protección del medio ambiente. Y si bien en su exposición de motivos hace tan solo una referencia implícita al Convenio de Aarhus - al reconocer "los avances alcanzados en los instrumentos internacionales y regionales" -, es evidente que tiene una estructura y contenido en gran parte similar a la de Convenio de Aahrús, articulándose en torno a los tres "derechos de acceso": el de información, el de participación y el acceso a la justicia (MÉDICI COLOMBO, 2018, p. 20-25).

Por otra parte, el texto del Acuerdo de Escazú refleja también las necesidades y particularidades de la región, en algunos puntos muy distintas de las del entorno europeo. Así, y entre otros aspectos novedosos, el Acuerdo de Escazú incorpora el compromiso de orientar y asistir a aquellas personas y grupos en situación de vulnerabilidad que encuentran especiales dificultades para ejercer con plenitud los derechos de acceso reconocidos en el Acuerdo (artículo 2.e y 4.5). Disposición esta que se adopta con el objetivo de contribuir a las desigualdades profundamente arraigadas en esa región que pueden menoscabar su aplicación. También incluye una disposición sobre el reconocimiento de los derechos de los "Defensores de los derechos humanos en asuntos ambientales" con el fin de garantizar "un entorno seguro y propicio en el que las personas, grupos y organizaciones 
que promueven y defienden los derechos humanos en asuntos ambientales puedan actuar sin amenazas, restricciones e inseguridad" (artículo 9). Se incorpora así una novedosa cláusula de indemnidad en beneficio de quienes actúen exigiendo el cumplimiento del derecho ambiental, con el objeto de protegerlos contra cualquier represalia por su actividad en defensa del medio ambiente - y de cuya aplicación en la práctica puede depender la del resto de las disposiciones del Acuerdo en un contexto socio-político como el de América Latina y el Caribe.

El derecho de participación pública se aborda en el artículo 7 del Convenio, que dispone con carácter general, en su apartado 1, que cada parte deberá "asegurar el derecho de participación del público" y, para ello, "se compromete a implementar una participación abierta e inclusiva en los procesos de toma de decisiones ambientales, sobre la base de los marcos normativos interno e internacional". Se trata de una obligación genérica cuyo contenido se va a concretar en los apartados que le siguen en los términos que examinamos a continuación.

En las disposiciones de este único artículo se regula tanto la participación en los procesos de toma de decisiones relativos a proyectos y actividades, y autorizaciones ambientales que tengan o puedan tener un impacto significativo sobre el medio ambiente (apartado 2); como la participación del público en procesos de elaboración de políticas, estrategias, planes, programas, normas y reglamentos (apartado 3). Ello a diferencia del Convenio de Aarhus, en el que el pilar sobre participación del público está integrado por tres artículos distintos: los artículos 6 a 8. En estas disposiciones el Convenio de Aarhus regula con distinto grado de precisión y exigencia los siguientes ámbitos: la participación en la adopción de decisiones relacionadas con determinadas actividades públicas o privadas (artículo 6); la participación en los planes, programas y políticas relativos al medio ambiente (artículo 7); y la participación en la elaboración de disposiciones reglamentarias o de instrumentos normativos jurídicamente obligatorios de aplicación general (artículo 8).

Cabe destacar, en primer lugar, que el Convenio de Aarhus incluye en su anexo I un listado exhaustivo de actividades con impacto en el medio ambiente en relación con las cuales en todo caso los Estados deben garantizar el derecho de participación conforme al artículo 6. Por el contrario, el Acuerdo de Escazú simplemente indica que se deberá garantizar la participación en aquellas actividades que tengan o puedan tener un "impacto significativo". Deja así indeterminados los supuestos 
en que en todo caso debe garantizarse el derecho de participación, y a expensas de lo que puedan precisar la legislación nacional, o los convenios internacionales en materias como evaluación de impacto ambiental o autorización de determinadas actividades. Los Estados que ratifiquen el Acuerdo de Escazú tendrán así un margen mucho más amplio de apreciación a la hora de decidir en qué supuestos es exigible la participación pública conforme a las previsiones que a continuación se exponen.

\subsection{Sobre las condiciones de la participación}

En los apartados 4 a 6 del artículo 7 del Acuerdo de Escazú se regulan las condiciones mínimas de participación que tienen que respetar cada Estado Parte del Acuerdo en relación con las decisiones ambientales a las que hacen referencia tanto su apartado 2 como 3. Condiciones que coinciden en gran medida con las establecidas en el Convenio de Aarhus en los apartados 3 a 8 del artículo 6 para las actividades específicas listadas en el Anexo I del Convenio (y que se aplican en este último Convenio solo parcialmente a la participación en planes, programas y políticas, por remisión del artículo 7 a los apartados 3, 4 y 7 del artículo 6).

Así, el apartado 4 del artículo 7 del Acuerdo de Escazú recoge el principio de participación temprana y eficaz, "desde las etapas iniciales del proceso de toma de decisiones, de manera que las observaciones del público sean debidamente consideradas". Principio que está redactado en términos análogos al artículo 6.4 del Convenio de Aarhus.

La obligación de establecer "plazos razonables" para la participación se establece en el apartado 5 del artículo 7, al igual que en el artículo 6.3 del Convenio de Aarhus.

Por su parte, el apartado 6 detalla la información mínima que deberá facilitarse al público "de forma efectiva, comprensible y oportuna, a través de medios apropiados" en el marco de cualquier procedimiento de participación: tipo o naturaleza de la decisión ambiental, autoridad responsable, procedimiento previsto para la participación con indicación de fecha y mecanismos previstos, y autoridades públicas. Se trata de exigencias en parte similares a las establecidas en el artículo 6.2 del Convenio de Aarhus, si bien el Convenio de Aarhus precisa que tal información se facilitará al "público interesado" (esto es, según el artículo 2.5 del Convenio de Aarhus, el que "resulta o pueda resultar afectado por las decisiones adoptadas" o "que tiene un interés que invocar en la toma 
de decisiones"), no al "público" en general, como dispone de forma más amplia el Acuerdo de Escazú (definido en su artículo 2.d como "una o varias personas físicas o jurídicas y las asociaciones, organizaciones o grupos constituidos por esas personas, que son nacionales o que están sujetos a la jurisdicción nacional del Estado Parte").

Por lo que respecta a los procesos de toma de decisiones relacionados con los proyectos y actividades a los que se refiere el apartado 2 del artículo 7 (los que tengan o puedan tener un "impacto significativo" sobre el medio ambiente), el apartado 17 del mismo artículo concreta de forma más detallada la información que ha de hacerse pública y que también incluye: a) la descripción del área de influencia y de las características físicas y técnicas del proyecto o actividad propuesto; b) la descripción de los impactos ambientales del proyecto o actividad y, según corresponda, el impacto ambiental acumulativo; c) la descripción de las medidas previstas con relación a dichos impactos; d) un resumen de los puntos anteriores "en lenguaje no técnico y comprensible"; e) los informes y dictámenes públicos de los organismos involucrados dirigidos a la autoridad pública vinculados al proyecto o actividad de que se trate; f) la descripción de las tecnologías disponibles para ser utilizadas y de los lugares alternativos para realizar el proyecto o actividad sujeto a las evaluaciones, cuando la información esté disponible.

Esta última disposición es, asimismo, en parte reflejo de las establecidas en el artículo 6.6 del Convenio de Aarhus, que establece un nivel de información mucho más preciso para las actividades y autorizaciones ambientales del anexo I del Convenio sometidas a evaluación de impacto ambiental y autorización (y que tampoco se aplica a las medidas de planificación o regulación). No obstante, el Convenio de Escazú incluye como novedad que se facilite información sobre las "acciones de monitoreo de la implementación y de los resultados de las medidas del estudio de impacto ambiental" (letra g), aspecto este esencial en el caso de los proyectos y actividades sometidas a este instrumento de tutela ambiental.

Finalmente el apartado 7 establece que el derecho a participar incluye la oportunidad de presentar observaciones por medios apropiados y disponibles, y que el resultado del proceso de participación será "debidamente tenidas en cuenta" por las autoridades en línea, de nuevo, con el artículo 6.8 del Convenio de Aarhus. 


\subsection{Sobre los resultados del proceso de participación}

Por lo que se refiere al resultado del proceso de participación, el Acuerdo de Escazú el apartado 8 del artículo 7 dispone que, una vez adoptada la decisión, el público sea "oportunamente informado de ella y de los motivos y fundamentos que la sustentan, así como del modo en que se tuvieron en cuenta sus observaciones". Por su parte, en este punto el artículo 6.9 del Convenio de Aarhus se limita a exigir que el público sea "rápidamente informado".

Además, el Acuerdo de Escazú incluye precisiones adicionales con respecto del Convenio de Aarhus: en primer lugar, establece que el público debe ser informado "del modo en que se tuvieron en cuenta sus observaciones"; y se añade en el apartado 9 la obligación de difusión de las decisiones resultantes a través de diversos medios, y que deberá incluir, asimismo, referencias a las acciones administrativas y judiciales pertinentes que puedan ejercer el público frente a las mismas. Precisiones estas que no recoge el Convenio de Aarhus.

\subsection{Principales singularidades y novedades del Convenio de Escazú}

El Convenio de Escazú incluye una serie de notables singularidades y novedades con respecto al Convenio de Aarhus que son fruto, en unos casos, de los retos específicos que se planean en Latinoamérica y el Caribe; en otros de los retos globales que se van a plantear en el momento en el que se negoció el Acuerdo y, por último, en algún punto de las carencias observadas a lo largo de los años en el Convenio de Aarhus.

Así, el Acuerdo Regional para América Latina y el Caribe prevé que las partes "promuevan", conforme a la legislación nacional, la "participación del público en foros y negociaciones internacionales en materia ambiental o con incidencia ambiental, de acuerdo con las reglas de procedimiento que para dicha participación prevea cada foro", así como la participación del público en instancias nacionales para tratar asuntos de foros internacionales ambientales. Aspecto éste sin duda de gran importancia - dada la creciente relevancia de los tratados y convenios internacionales y regionales en el desarrollo de las políticas y del Derecho ambiental nacional -, y que no aborda el Convenio de Aarhus.

El Convenio de Escazú presta asimismo especial atención a la diversidad de los países de la región, imponiendo a las Partes la obligación 
de establecer las condiciones propicias para que la participación pública se adecúe a las características sociales, económicas, culturales, geográficas y de género del público (apartado 10), así como idiomáticas (apartado 11). Debe además valorarse "el conocimiento local, el diálogo y la interacción de las diferentes visiones y saberes, cuando corresponda" (apartado 13).

Por otra parte, exige no sólo que a las autoridades públicas se esfuercen por identificar y facilitar la participación del "público directamente afectado por proyectos y actividades que tengan o puedan tener un impacto significativo sobre el medio ambiente", sino que atiende también en especial a la participación de "personas o grupos en situación de vulnerabilidad", y al respeto de los derechos de los pueblos indígenas y comunidades locales.

Finalmente, el Acuerdo de Escazú prevé expresamente que cada Parte "alentará el uso de las nuevas tecnologías de la información, y la comunicación, tales como los datos abiertos, en los diversos idiomas usados en el país, cuando corresponda", al tiempo que prevé que los medios electrónicos "serán utilizados de una manera que no generen restricciones o discriminaciones para el público" (artículo 4.9). Hace así referencia, por una parte, a las posibilidades y facilidades que las nuevas tecnologías ofrecen para articular los procesos de participación, al tiempo que a la necesidad de evitar que la brecha digital pueda dejar fuera de los procesos de participación a aquellas personas que no tienen fácil acceso a las mismas, acrecentando así las profundas desigualdades que aún existen en la región.

Este Acuerdo, que se encuentra abierto a la adhesión de todos los países de esta región incluidos en el anexo I, ha sido firmado ya por 16 estados de América Latina y el Caribe (entre los que se encuentra Argentina, Bolivia, Brasil, Costa Rica, Ecuador, Guatemala, México, Panamá, Paraguay, Perú, República Dominicana y Uruguay), y entrará en vigor, conforme a su artículo 22, a partir de la fecha en que sea depositado el undécimo instrumento de ratificación o adhesión. Se trata, sin duda, de un paso fundamental hacia la materialización del principio 10 de la Declaración de Río a escala global, que se suma al dado con el Convenio de Aarhus hace ya dos décadas. 


\section{NOVEDADES NORMATIVAS EN ESPAÑA. LOS RETOS PENDIENTES}

El Convenio de Aarhus, y las Directivas adoptadas por la UE en su marco, impulsaron en España la adopción de la Ley 27/2006, de 18 de julio, por la que se regulan los derechos de acceso a la información, de participación pública y de acceso a la justicia en materia de medio ambiente (en adelante Ley 27/2006). Esta Ley, que adecúa la legislación básica española a las obligaciones asumidas al ratificar el Convenio declara en su Exposición de Motivos que tiene por objeto articular "los medios instrumentales adecuados" para que los ciudadanos, individual y colectivamente, puedan participar efectivamente de la adopción de medidas dirigidas a garantizar lo dispuesto en el artículo 45 de nuestra Constitución: el derecho de todos a disfrutar de un medio ambiente adecuado para el desarrollo de la persona, así como el deber de conservarlo, mediante la colaboración activa de la sociedad en el cumplimiento del mandato dirigido a los poderes públicos de defender y restaurar el medio ambiente, apoyándose en la indispensable solidaridad colectiva. Participación que consagra nuestra Constitución con carácter general en el artículo 9.2, y en el artículo 105 para el ámbito administrativo en particular, como cauce para una mayor transparencia y acierto en la gestión de los asuntos públicos, y de funcionamiento democrático de la sociedad (PLAZA MARTÍN, 2018, p. 5).

Más de una década después, la novedad más significativa en materia de participación ambiental en el marco normativo español es la adopción de La Ley 9/2018, de 5 de diciembre que modifica entre otras normas ambientales la Ley 21/2013 de evaluación ambiental con el objetivo de adecuar plenamente la legislación básica española a la normativa de la Unión Europea (en concreto, a las modificaciones que en su momento introdujo la Directiva 2014/52/UE en la Directiva 2011/92/UE, del Parlamento Europeo y del Consejo, de 13 de diciembre de 2011, relativa a la evaluación de las repercusiones de determinados proyectos públicos y privados sobre el medio ambiente).

No obstante, y como veremos en los siguientes apartados, sigue pendiente el impulso de medidas que den una respuesta a los principales retos a los que se enfrente la participación pública ambiental en España. 


\subsection{La "novedad": la utilización preferente de los medios electrónicos}

En materia de participación se modifican diversas disposiciones de la Ley 21/2013 (los artículos 9.3, 21.4, 22.1, 28.4, 36.3 y 37.3) para implantar la utilización preferente de los medios electrónicos con el fin de "garantizar la participación efectiva de las personas interesadas en los procesos de evaluación ambiental". Se incluye así, entre las obligaciones generales en materia de evaluación ambiental (artículo 9), un apartado tercero en el que se dispone que los trámites de información pública, y de consulta a las Administraciones Públicas afectadas y a las personas interesadas regulados en esta ley, "se efectuarán por vía electrónica y mediante anuncios públicos u otros medios apropiados que garanticen la máxima difusión a la ciudadanía dentro de los municipios afectados y los colindantes". Se impone también a todas las Administraciones Públicas la obligación de adoptar las medidas necesarias para garantizar, dentro del trámite de información pública, que la información pertinente sea accesible electrónicamente por parte del público a través de, al menos, un portal central o de puntos de acceso sencillo, en el nivel de la administración territorial correspondiente.

La exigencia de que las Administraciones Públicas garanticen que la documentación que debe someterse a información pública tenga la máxima difusión entre el público, utilizando los medios electrónicos, se replica después en diversas disposiciones de la Ley: en los arts. 21.4, 22.1 y 28.4 en relación con la evaluación ambiental estratégica; y en los arts. 36.3 y 37.3 en relación con la evaluación de impacto ambiental estratégico. Conforme a su Exposición de motivos, estas modificaciones tienen "la finalidad de reforzar el acceso público a la información y la transparencia”.

Asimismo, y en relación con la notificación a las personas interesadas y que deban ser consultadas en el marco de los procedimientos de evaluación de impacto ambiental de proyectos, el apartado cuarto del artículo 9 refuerza las vías de notificación cuando dichas personas sean desconocidas, exigiendo que se lleve a cabo (además de mediante anuncio en el "Boletín Oficial del Estado" o diario oficial correspondiente), mediante la publicación de anuncios en el tablón de edictos, y en su caso, en la página web de los Ayuntamientos afectados. Establece a tales efectos el plazo mínimo de exposición (treinta días hábiles) y, más peculiar y novedoso aún, establece un "mecanismo de control" de dicha exposición, 
al exigir que una vez transcurrido el plazo los ayuntamientos remitan al órgano sustantivo o, en su caso, al órgano ambiental, "un certificado de exposición pública en el que haga constar el lugar y periodo en que ha estado expuesta la documentación ambiental".

\subsection{Valoración: los retos pendientes}

Hoy día resulta sin duda imprescindible garantizar al público en general, a las personas interesadas y a las organizaciones ambientales, un fácil acceso a la información a través de medios electrónicos, así como la posibilidad de hacer llegar a las Administraciones sus observaciones y alegaciones en los procedimientos de evaluación ambiental por los mismos medios. Esta es, sin embargo, una modificación normativa que, por las razones que se exponen a continuación, supone un modesto avance en la práctica, y cuya aplicación tiene que llevarse a cabo sin menoscabo en ningún momento de los grupos de población que no tengan fácil acceso a dichos medios.

En primer lugar, tales medios ya venían siendo utilizados de facto por gran parte de las Administraciones Públicas, según se recoge en los informes de cumplimiento presentados por España a las reuniones de las Partes del Convenio de Aarhus: en dichos informes se da cuenta - entre otros aspectos - de las prácticas administrativas relacionadas con el uso de las nuevas tecnologías para facilitar la transparencia y la participación temprana, destacando en particular la publicación en las páginas web de los órganos ambientales, fundamentalmente estatal y autonómicos, de información sobre los distintos procesos de participación. Más en concreto, y dentro del procedimiento de evaluación ambiental - tanto de planes y programas como de proyectos - la Administración General del Estado vienen poniendo a disposición del público la documentación ambiental, y facilitando información sobre la tramitación telemática de los expedientes a través de la página web del Ministerio responsable en materia de medio ambiente (vid. por ej., el Informe de Cumplimiento presentado por España a la Sexta Reunión de las partes del Convenio celebrada en Riga, Montenegro 11-14 de septiembre 2017, p. 28, apartado 111). No deja de ser cierto, por otra parte, que su introducción en la Ley supone la transformación de una práctica administrativa en una obligación legal para todas las Administraciones Públicas, lo que sin duda supone una mejora en el marco normativo existente hasta el momento. 
En segundo lugar, están aún pendientes de abordar las principales carencias y retos a los que se enfrenta en España la consecución de una participación pública realmente "efectiva" en materia de medio ambiente, conforme exige el Convenio de Aarhus. Carencias y problemas que se reflejan - al menos una parte de ellas - tanto en los informes de aplicación de dicho Convenio elaborados por España, ya citados, como en las resoluciones adoptadas en relación con nuestro país por el Comité de Cumplimiento del Convenio de Aarhus y la Conferencia de las Partes. Se esbozan a continuación algunos de ellos:

- Plazos suficientes para la participación: En el Informe de aplicación presentado por España a la Sexta Reunión de las Partes del Convenio (2017) se afirma que "el plazo mínimo regulado en las legislaciones sectoriales para presentar alegaciones en los procedimientos sujetos a intervención ambiental, especialmente en las EIA [Evaluación de Impacto Ambiental] y AAI [Autorización Ambiental Integrada] resulta, a juicio de algunos ciudadanos e interlocutores sociales, insuficiente dado lo voluminoso de los expedientes y su complejidad técnica" (pág. 26, apartado 119). Cuestión esta que ha sido denunciada por ONGs ambientales ante el Comité de Cumplimiento del Convenio de Aarhus en diversas ocasiones, el cual ha emitido diversas declaraciones y recomendaciones al respecto, dando lugar finalmente en la Cuarta Reunión de las Partes (2011) a la adopción de la Decisión IV/f sobre el cumplimiento por parte de España de las obligaciones del Convenio. En dicha Decisión -se aborda, por una parte, diversos incumplimientos de disposición del Convenio por parte del Ayuntamiento de Murcia durante la tramitación de un proyecto de urbanización de zona residencial; y, por otra, la Administración Extremeña y la Administración General del Estado en el marco de los procedimientos para autorizar la instalación de tres centrales térmicas de ciclo combinado y una refinería de petróleo en Extremadura. En la misma la Cuarta Reunión de la Partes constataba que aún no se habían adoptado todas las medidas necesarias para poner fin al incumplimiento de las diversas disposiciones del Convenio, entre ellas las relativas a la participación del público, reiterando dicha Decisión la necesidad de "concienciar a las administraciones y a las personas al servicio de las mismas para establecer plazos razonables de participación pública en los procesos de toma de decisiones" (ECE, Decision V/9k on compliance by Spain, 1 de julio de 2011, ECE/ MP.PP/2011/CRP.8, apartado 6). 
- Las limitaciones de las comunicaciones a través de las nuevas tecnologías: Siendo sin duda imprescindible facilitar la participación a través de las nuevas tecnologías de la información, es también fundamental compaginarlas debidamente con otros instrumentos de comunicación convencionales, y con mecanismos y actuaciones que garanticen que la brecha digital no menoscabe las posibilidades de participación de aquellos segmentos de población que no tengan fácil acceso a los mismos. Nótese que, en relación con esta cuestión, el Comité de Cumplimiento del Convenio de Aarhus ha declarado que cuando el texto de la decisión finalmente adoptada por una Administración tras un proceso de participación (en este caso se trataba de la tramitación de una autorización por la Generalitat de Cataluña) se publica únicamente en la web del Departamento responsable de medio ambiente se vulnera el artículo 6.9 (información al público una vez adoptada la decisión siguiendo procedimiento apropiado). En relación con dicha infracción, el Comité ha recomendado al Estado español adoptar las medidas legislativas, reglamentarias o de otro tipo necesarias para asegurar que el público sea informado rápidamente de la decisión finalmente adoptada no solo a través de internet, sino también de otros medios, incluyendo - pero no necesariamente solo los utilizados para informar al público cuando se inicie un proceso de toma de decisiones conforme al párrafo 2 del artículo 6 (vid. UNECE, Compliance Committee, Findings and recommendations with regard to communication ACCC/C/2014/99 concerning compliance by Spain, ECE/MP.PP/C.1/2017/17, p. 17). Incumplimiento éste que fue también puesto de manifiesto en la Sexta Reunión de las Partes del Convenio (Decision VI/8j Compliance by Spain with its obligations under the Convention, Excerpt from the addendum to the report of the sixth session of the Meeting of the Parties, ECE/MP.PP/2017/2/Add.1, Montenegro 11-13 septiembre).

- Escasa participación en la práctica: está por ver si la facilidad de comunicación a través de medios electrónicos con la Administración coadyuva en la práctica a una mayor implicación y participación de la sociedad en estos procesos. En todo caso, para abordar este problema, que se identifica sistemáticamente en los Informes del Gobierno de España (vid. por ejemplo el Informe de 2017 ya citado, pág. 28, apartado 128) es necesario articular nuevas medidas proactivas por parte de las Administraciones, explorando otros cauces que impulsen 
una interacción real entre los ciudadanos y la Administración desde las fases iniciales de la del proceso de toma de decisión, que vayan más allá de los trámites que hasta el momento se vienen utilizando tradicionalmente (información pública y consulta a los interesados), y que impulsen una verdadera cultura de participación (vid. VICENTE DAVILA, 2019, p. 8-10).

\section{LA APLICACIÓN JUDICIAL DE LAS DISPOSICIONES SOBRE PARTICIPACIÓN PÚBLICA EN MATERIA DE MEDIO AMBIENTE}

La aplicación de las disposiciones del Convenio de Aarhus en materia de participación pública por los Tribunales plantea cuestiones de especial interés cuando las decisiones sobre el medio ambiente se adoptan a través de un procedimiento compuesto, en el que intervienen no sólo la Administración española, sino también instituciones de la Unión Europea. No obstante, los tribunales nacionales vienen controlando con normalidad el cumplimiento de las disposiciones del Convenio de Aarhus sobre participación pública. Participación que se la jurisprudencia considera como un acto de trámite cualificado cuya omisión va a conllevar la nulidad de la medida o plan que se adopte en vulneración de las mismas.

\subsection{Los procedimientos compuestos en que intervienen la Administración nacional y la Unión Europea}

Entre las decisiones judiciales en las que a lo largo de 2018 se han aplicado disposiciones sobre participación pública en materia de medio ambiente destaca, en particular, la Sentencia de la Sala de lo Contencioso-Administrativo Tribunal Supremo (Sección Quinta) núm. 1203/2018 de 12 julio. Esta resolución plantea problemas de especial interés en relación con el cumplimiento del artículo 7 del Convenio de Aarhus durante la tramitación por el Gobierno español del Plan Nacional Transitorio para grandes instalaciones de combustión (PNT), adoptado conforme a la Directiva 2010/75/UE sobre las emisiones industriales (prevención y control integrados de la contaminación).

La Sentencia desestima el recurso contencioso-administrativo interpuesto por la Asociación Instituto Internacional de Derecho y 
Medioambiente (IIDMA) contra el Acuerdo del Consejo de Ministros de 25 de noviembre de 2016, por el que se aprueba el Plan Nacional Transitorio para grandes instalaciones de combustión (PNT). Se reprochaba a dicho Acuerdo, entre otros vícios, haber eludido las exigencias de participación pública previstas en la Ley 21/2013 de evaluación ambiental y en el artículo 7 del Convenio de Aarhus.

La adopción de dicho Plan permite, conforme al artículo 32 de la Directiva 2010/75/UE, que durante su período de aplicación (del 1 de enero de 2016 al 30 de junio de 2020) las instalaciones cubiertas por el mismo queden exentas del cumplimiento de los valores límite de emisión que les correspondería según el artículo 30, apartado 2, de la misma. Conforme al apartado 5 del artículo 32 de la Directiva, a más tardar el 1 de enero de 2013, los Estados miembros tenían que comunicar a la Comisión sus PNTs para su evaluación, y si la Comisión "no plantea objeciones dentro de los doce meses siguientes a haberlo recibido, el Estado miembro de que se trate lo considerará aprobado". También debe informarse a la Comisión de todo cambio posterior del plan.

La IIDMA alegaba en su recurso que sólo la tercera versión del PNT fue sometida a un procedimiento de consulta pública, y únicamente por un período de 21 días transcurridos del 4 al 21 de diciembre de 2015.

En su sentencia el Tribunal Supremo puso de manifiesto que el procedimiento de tramitación de dicho Plan venía determinado por la aplicación del artículo 32.5 de la Directiva 2010/75/UE, según el cual tales planes han de ser comunicados a la Comisión para su aprobación (y comunicarse igualmente cualquier modificación del mismo posterior). Da cuenta así que de que una vez que España decidió acogerse a un Plan Nacional Transitorio en aplicación de la Directiva, envió a la Comisión Europea una primera propuesta de Plan el 14 de diciembre de 2012, que la Comisión no aprobó por considerar que era necesario modificar en varios puntos para ajustarse a los requisitos establecidos en la Directiva 2010/75/ UE (Decisión de la Comisión 2013/799/UE, de 17 de diciembre, relativa a la notificación por parte del Reino de España del plan nacional transitorio a que se refiere el artículo 32 de la Directiva 2010/75/UE del Parlamento Europeo y del Consejo, sobre las emisiones industriales).

El segundo borrador remitido por el Gobierno español con las debidas modificaciones no fue ya objeto de objeciones; no obstante, debido a que el listado definitivo de las instalaciones acogidas al Plan se modificó en noviembre de 2015 (dado que algunas de las plantas inicialmente acogidas 
a dicho plan decidieron renunciar al mismo), se volvió a notificar a la Comisión el texto el 20 de noviembre de 2015. Ese tercer borrador fue el que finalmente se sometió a participación pública por un plazo que iba desde el 4 hasta el 21 de Diciembre del 2015, ambos inclusive, por ser, según alegó la Administración, "la versión que se consideró más actualizada puesto que se ajustaba a aquélla sobre la cual la Comisión no había formulado observaciones".

El Tribunal Supremo rechazó en su sentencia que este modo de proceder vulnerase las disposiciones del Convenio de Aarhus, constatando que "el PNT no sólo se sometió a información pública a través de la publicación en la página web del Departamento, sino que también se tuvieron en cuenta los comentarios y observaciones realizadas por los distintos órganos a los que se remitió el Plan para su participación" (FD 12).

En su motivación argumenta, además, que "el convenio Aarhus no se refiere a un plazo concreto para el trámite de la consulta pública, dejándolo a elección de los Estados Miembros, por lo que se puede concluir que el trámite de participación pública del PNT se realizó de conformidad con la Ley 27/2006, de 18 de julio, por la que se regulan los derechos de acceso a la información, de participación pública y de acceso a la justicia en materia de medio ambiente, que transpone a nuestro ordenamiento jurídico dicho Convenio".

Por último, y en relación con el principio de participación temprana, el Tribunal Supremo declaró que "el Convenio Aarhus establece que la participación del público debe realizarse cuando la misma pueda ejercer una influencia real y dificilmente éste hubiera podido ejercerla sobre un borrador que no tenía ni siquiera el visto bueno de la Comisión".

Dejando de lado la cuestión de si el plazo fue o no razonable (para lo cual habría que ponderar la complejidad del Plan y el número de días otorgado para su análisis - que precisa en todo caso de conocimientos expertos - y para realizar las consiguientes alegaciones), la principal cuestión que a mi juicio plantea esta sentencia está en relación el último argumento que esgrime el Tribunal para descartar que no se hubiera respetado en el curso del procedimiento la obligación de posibilitar que la participación tuviera lugar de forma "temprana" y cuando el público pueda ejercer una influencia real, conforme dispone al apartado 2 del artículo 6 del Convenio de Aarhus.

En efecto, el artículo 6.2 del Convenio de Aarhus (aplicable también a los planes y programas por remisión al mismo en el artículo 7) exige 
que "la participación del público comience al inicio del procedimiento, es decir, cuando todas las opciones y soluciones sean aún posibles y cuando el público pueda ejercer una influencia real”. En línea con lo establecido por el Convenio de Aarhus, el artículo 16.1 de la Ley 27/2006, de 18 de julio, por la que se regulan los derechos de acceso a la información, de participación pública y de acceso a la justicia en materia de medio ambiente, exige en relación con los planes y programas que las Administraciones Públicas, al establecer o tramitar los procedimientos que resulten de aplicación, velarán porque "b) El público tenga derecho a expresar observaciones y opiniones cuando estén abiertas todas las posibilidades, antes de que se adopten decisiones sobre el plan, programa o disposición de carácter general".

Sin embargo, cuando estamos ante un procedimiento administrativo compuesto como es el de este caso, en el que la Comisión Europea debe aprobar las medidas propuestas por un Estado miembro, ¿se puede entender que se respeta el artículo 7 en relación con el artículo 6.4 del Convenio una vez que se ha presentado un borrador de Plan a la Comisión y este ha sido aprobado por dicha institución de la UE? En mi opinión, en el momento en el que la Comisión aprueba un plan en el que se han incorporado las observaciones y objeciones previamente hechas al mismo por la esta institución europea, y aunque esté pendiente aún la aprobación formal definitiva por la Administración nacional competente, difícilmente cabe entender estén "abiertas todas las opciones y soluciones" - o al menos "suficientemente abiertas" como para que el público pueda ejercer "una influencia real".

En el supuesto del PNT, en particular, hay que tener en cuenta también que dicho Plan tenía vocación de ser aplicado desde el 1 de enero de 2016, y que cualquier modificación sustancial introducida como resultado del proceso de consulta hubiera tenido que volver a ser comunicada a la Comisión para su aprobación. Es evidente que la participación del público no comenzó "al inicio del procedimiento", y las circunstancias y factores que aquí hemos destacamos ponen claramente en cuestión, en mi opinión, que estuvieran abiertas en ese momento "todas las posibilidades" antes de su aprobación definitiva, conforme exige el artículo 7 del Convenio.

En definitiva, parece lógico concluir que en el marco de procedimientos compuestos en los que operan tanto la Administración nacional como la Comisión Europea en la toma de decisión, y en particular cuando esta última tiene que aprobar medidas propuestas por las autoridades nacionales, el principio de participación temprana consagrado en el 
artículo 6.2 del Convenio de Aarhus exige que el proceso de participación se lleve a cabo antes de que la Administración nacional comunique a las instancias europeas, para su aprobación, el plan o las medidas que deben o pueden adoptarse en ejecución de una norma de la Unión Europea. Ello sin perjuicio de los cambios que posteriormente, por exigencia del Derecho de la Unión, pueda requerir la Comisión Europea. De otro modo, el público no tendrá apenas margen alguno de influencia mediante su participación en el proceso.

\subsection{Otros casos: la "normalidad" en la aplicación judicial de las disposiciones sobre participación pública}

Por último, haremos breve mención a dos resoluciones dictadas por Tribunales Superiores de Justicia que son ejemplo de cómo se viene ejerciendo el control judicial sobre el cumplimiento las disposiciones nacionales o autonómicas sobre participación pública a través de las que se da aplicación al segundo pilar del Convenio de Aarhus: (i) Una primera en la que se declara la nulidad de pleno derecho de la decisión adoptada por la Administración por omitir los trámites de participación pública establecidos por el Real Decreto 815/2013 por el que se aprueba el Reglamento de emisiones industriales; (ii) la segunda va a desestimar el recurso presentado por una asociación de vecinos contra el Plan Acústico Municipal aprobado por el Municipio de Elche tras declarar que la Administración cumplió con las disposiciones de participación pública establecidas en la normativa específica en materia de ruido.

(i) El Tribunal Superior de Justicia de Galicia (Sala de lo Contencioso-Administrativo, Sección 2a ) estimó en su Sentencia num. 370/2018 de 28 de junio el recurso interpuesto por el Ayuntamiento de Pontevedra contra la Resolución de 22 de agosto de 2016 dictada por la Consellería de Medio Ambiente e Ordenación do Territorio, por la que se modificó el plazo de vigencia de la autorización ambiental integrada otorgada a Electroquímica del Noroeste, SAU para la producción de productos químicos de la industria cloro-alcalí por electrolisis. El Ayuntamiento basó su recurso en la vulneración del procedimiento legalmente establecido para proceder a la modificación temporal operada, porque no se concedió el trámite de audiencia al Ayuntamiento de Pontevedra y tampoco se abrió trámite alguno de información pública. El Tribunal 
declara la resolución de la Consellería nula de pleno derecho, conforme al artículo 62.2 de la LPAC - entonces vigente - porque "resultaron preteridos, entre otros, los tramites de información pública y de alegaciones y audiencia" (en ese caso regulados en el artículo 16 del Real Decreto 815/2013 por el que se aprueba el Reglamento de emisiones industriales y de desarrollo de la Ley 16/2002, de 1 de julio, de prevención y control integrados de la contaminación).

(ii) Por el contrario, el Tribunal Superior de Justicia de la Comunidad Valenciana (Sala de lo Contencioso-Administrativo, Sección $1^{\text {a }}$ ) desestimó en su Sentencia núm. 232/2018 de 29 marzo el recurso planteado por la Asociación de Afectados por el Ruido de Elche contra los acuerdos del Ayuntamiento de Elche de 23 de diciembre de 2013 por el que se aprobó el Mapa Estratégico del Ruido de Elche y el Plan de Acción de Contaminación Acústica del municipio; y el acuerdo de 30 de junio de 2014 que aprobó definitivamente el Plan Acústico Municipal (PAM). La Asociación alegaba, en primer lugar, que durante la elaboración del plan acústico municipal el Ayuntamiento no dio cumplimiento a las garantías de participación ambiental establecidas en el Decreto 97/2010, de 11 de junio, del Consell, por el que se regula el ejercicio del derecho de acceso a la información ambiental y de participación pública en materia de medio ambiente de la Comunitat Valenciana.

En su Sentencia el Tribunal constata que la participación en este ámbito se rige - por remisión del Propio Decreto 97/2010 - por la normativa específica en materia de ruido (el artículo 24 de la Ley 7/2002 de la Generalitat e Protección contra la Contaminación Acústica, y el artículo 15 del Decreto 104/2006, del Consell de planificación y gestión en materia de contaminación acústica), y que la Administración se atuvo a la misma en la tramitación y aprobación del PAM objeto del recurso: dictó acuerdo sometiendo a información pública el plan; lo expuso en la web municipal por periodo de un mes; publicó edictos en un diario de información general de gran difusión en la provincia de Alicante; publicó edictos en el diario oficial de la Generalitat; y confirió trámite de audiencia a las principales asociaciones afectadas por el plan acústico municipal, entre ellas a la ahora recurrente, Asociación de Afectados por el Ruido de Elche, que formuló alegaciones en dos ocasiones $\left(\mathrm{FD} 2^{\circ}\right)$. En segundo lugar, rechaza la alegación según la cual el PAM debió haberse sometido a un segundo trámite de información tras las modificaciones introducidas por el Ayuntamiento a 
raíz del informe de la Dirección General de Calidad Ambiental - y que la Asociación considera de gran importancia y envergadura. El Tribunal declara a tales efectos que las disposiciones aplicables establecen un único trámite de información pública en la aprobación de los proyectos de plan acústico municipal; a mayor abundamiento, precisa que las modificaciones introducidas por el Ayuntamiento tras el informe emitido por la DG de Calidad Ambiental no eran modificaciones sustanciales, y que de las mismas tuvo pleno conocimiento la Asociación de Afectados por el Ruido de Elche, que tuvo oportunidad - y no lo hizo - de formular alegaciones antes de la aprobación definitiva del PAM corregido.

Tal pronunciamiento parece dejar abierta la puerta a que, en aquellos casos en que se produzcan modificaciones sustanciales de tal cariz que puedan poner en entredicho la efectividad del proceso de participación pública. En relación con esta cuestión es necesario recordar la doctrina sobre participación pública establecida por el Tribunal Constitucional en su Sentencia 28/2018, de 16 de febrero, en la que declara que

La información pública no es un mero trámite en el procedimiento de elaboración de los planes, sino uno esencial por la especial incidencia que tienen los planes urbanísticos en la vida de los ciudadanos. De ello resulta la correlativa necesidad de asegurar el derecho a la participación pública en el planeamiento urbanístico, que resulta de la regla estatal - y que, obviamente ha de ser efectiva -, derecho que se vulnera si se aprueba el instrumento de planeamiento sin el correspondiente trámite de información pública, $y$, también, cuando se han introducido, sin ese trámite, modificaciones sustanciales en el planeamiento aprobado. En ese último caso la participación tampoco es efectiva, en cuanto referida a un plan que no es el que contiene las decisiones que la Administración se propone adoptar en el instrumento de planeamiento y sobre las que ha de permitir que se exprese la opinión ciudadana.

Dicha declaración, si bien se pronuncia en relación con la elaboración de planes urbanísticos, es sin duda igualmente aplicable en el ámbito de la planificación o decisión ambiental, y fundamental para garantizar la aplicación eficaz de las disposiciones del Convenio de Aarhus en nuestro Estado. Estas decisiones judiciales muestran, sin duda, la importancia que nuestro ordenamiento jurídico y nuestros tribuales otorgan a estos derechos procedimentales para la protección del medio ambiente. 


\section{CONCLUSIONES}

El Convenio de Aarhus, siendo un convenio internacional regional, no solo está impulsando la democracia ambiental en los países del continente europeo que lo han ratificado, sino que también está sirviendo de referente en otras regiones de nuestro planeta para la adopción de instrumentos internacionales similares. El caso más destacado es el del Acuerdo regional sobre el acceso a la información, la participación pública y el acceso a la justicia en asuntos ambientales en América Latina y el Caribe, firmado el 4 de marzo de 2018 en la ciudad costarricense de Escazú con el apoyo de la Comisión Económica de las Naciones Unidas para América Latina y el Caribe (CEPAL). Este último Acuerdo, además de extender estos derechos en defensa del medio ambiente a una importantísima región de nuestro planeta, ha introducido algunas mejoras y precisiones con respecto a las inicialmente establecidas en el Convenio de Aarhus, en ámbitos como la participación a través de las nuevas tecnologías, o la igualdad de acceso al derecho de participación. El Convenio de Aarhus, por otra parte, establece obligaciones más precisas en relación con la obligación de someter a participación pública las actividades susceptibles de tener un especial impacto en el medio ambiente enumeradas en su Anexo I.

Por lo que se refiere a la legislación española, el Convenio de Aarhus ha supuesto, sin duda, un importante avance en el Derecho español en materia de participación pública y de protección del medio ambiente. Las últimas modificaciones de la normativa básica estatal ha venido de la mano, sin embargo, de la modificación de la Directiva de la Unión Europeo sobre Evaluación de Impacto Ambiental. Modificaciones que se limitan fundamentalmente a incorporar la obligación de las Administraciones de facilitar la participación pública a través de las nuevas tecnologías de la información y la comunicación. No obstante, todavía subsisten importantes carencias y retos para alcanzar una participación

Sin embargo, la adopción de normas adecuadas no basta para lograr una participación ciudadana "real y efectiva". Los propios informes de cumplimiento presentados por España a las reuniones de las Partes del Convenio de Aarhus ponen de manifiesto que la participación pública en estos procedimientos sigue siendo muy "escasa", y las decisiones adoptadas por el Comité de Cumplimiento del Convenio en relación con España, evidencian todavía ámbitos en donde es necesario incrementar el esfuerzo para cumplir debidamente con las obligaciones asumidas con el 
Convenio. Junto con la adopción de un marco normativo adecuado para responder a las obligaciones contraídas por el Estado español con la firma y ratificación del Convenio, es necesario seguir avanzando en la formación, sensibilización e implicación no sólo de toda la ciudadanía, sino de las distintas Administraciones, en un modelo de desarrollo sostenible.

Por último, la tutela judicial efectiva de este derecho de participación es esencial a tal fin, y a esta tarea contribuyen en general los Tribunales de forma decidida.

\section{REFERENCIAS}

BAN KI-MOON. Foreword. In: UNECE - UNITED NATIONS ECONOMIC COMMISSION FOR EUROPE. The Aarhus Convention: an implementation guide. 2. ed. Genève: United Nations, 2014.p. 3. Disponível em: <http://www.unece.org/fileadmin/DAM/env/pp/Publications/Aarhus Implementation_Guide_interactive_eng.pdf $>$. Acesso em: 19 jul. 2019.

BÁRCENA, A. Prefacio. In: NACIONES UNIDAS. Acuerdo Regional sobre el Acceso a la Información, la Participación Pública y el Acceso a la Justicia en Asuntos Ambientales en América Latina y el Caribe. Santiago: Cepal, 2018. p. 7-9.

GÓMEZ PEÑA, N. El arduo camino hacia la implementación de la democracia ambiental en América Latina y el Caribe. In: FARN FUNDACIÓN AMBIENTE Y RECURSOS NATURALES. Informe Ambiental Anual 2018, Buenos Aires: FARN, 2018. p. 365-380. Disponível em: $<$ https://www.iafonline2018.com/cap4>. Acesso em: 19 jul. 2019.

MÉDICI COLOMBO, G. El Acuerdo Escazú: la implementación del Principio 10 de Río en América Latina y el Caribe. Revista Catalana de Dret Ambiental, v. 9, n. 1, p. 1-66, 2018.

OLMOS GIUPPONI, B. Fostering environmental democracy in Latin America and the Caribbean: An analysis of the Regional Agreement on Environmental Access sights. Review of European, Comparative and International Environmental Law, v. 28, n. 2, p. 1-16, 2019.

ORSO BORILE, G. Delineando a democracia ambiental: apontamientos sobre a participação popular e o Acordo de Escazú. Revista Catalana de Dret Ambiental, v. 9, n. 1, p. 1-17, 2018. 
PLAZA MARTÍN, C. La aplicación de las disposiciones del segundo pilar del Convenio de Aarhus en España. Los avances y los viejos retos. Revista Catalana de Dret Ambiental, v. 9, n. 1, p. 1-70, 2018.

PRIEUR, M. La participation d'Aarhus, instrument universel de la démocratie environnementale. Revue Juridique de l'Environnement, $\mathrm{n}$. spécial, p. 9-29, 1999.

VICENTE DAVILA, F. La Ley 9/2018 por la que se modifica la Ley 21/2013 de evaluación ambiental: otra oportunidad perdida para avanzar hacia una participación real y efectiva. Actualidad Jurídica Ambiental, n. 86, Sección “Comentarios de legislación”, 21 de enero de 2019.

Artigo recebido em: 10/05/2019.

Artigo aceito em: 19/07/2019.

\section{Como citar este artigo (ABNT):}

PLAZA MARTÍN, C. Protección del medio ambiente y participación pública: la reciente evolución a nivel internacional y en España. Veredas do Direito, Belo Horizonte, v. 16, n. 35, p. 11-36, maio/ago. 2019. Disponível em: $\quad<$ http://www.domhelder.edu.br/revista/index.php/veredas/article/ view/1543>. Acesso em: dia mês. ano. 